Supporting Information

\title{
Formation of large crystalline domains in semiconducting polymer with semi-fluorinated alkyl side chains and application to high-performance thin-film transistors
}

Youn-Jung Heo ${ }^{1, \dagger}$, Hyung-Gu Jeong ${ }^{2, \dagger}$, Jihong Kim ${ }^{3}$, Bogyu Lim ${ }^{4}$, Juhwan Kim ${ }^{5}$, Yunseul Kim ${ }^{1}$, Boseok Kang ${ }^{6}$, Jin-Mun Yun ${ }^{7}$, Kilwon Cho ${ }^{6}$ and Dong-Yu Kim ${ }^{1, *}$

${ }^{1}$ Heeger Center for Advanced Materials, School of Materials Science and Engineering, Gwangju Institute of Science and Technology (GIST), Gwangju 61005, Republic of Korea

${ }^{2}$ Busan Institute of S\&T Evaluation and Planning (BISTEP), Busan 48058, Republic of Korea

${ }^{3}$ Korea Institute of S\&T Evaluation and Planning (KISTEP), Seoul 06775, Republic of Korea

${ }^{4}$ Korea Research Institute of Chemical Technology (KRICT), Ulsan 44412, Republic of Korea

${ }^{5}$ Future Technology Research Center, Corporate R\&D, LG Chem Research Park, Seoul 07796, Republic of Korea

${ }^{6}$ Department of Chemical Engineering, Pohang University of Science and Technology, Pohang 37673, Korea

${ }^{7}$ Radiation Research Division for Industry and Environment, Korea Energy Research Institute (KAERI), Jeongeup 56212, Republic of Korea.

E-mail: kimdy@ gist.ac.kr

$\dagger$ These two authors contributed equally 


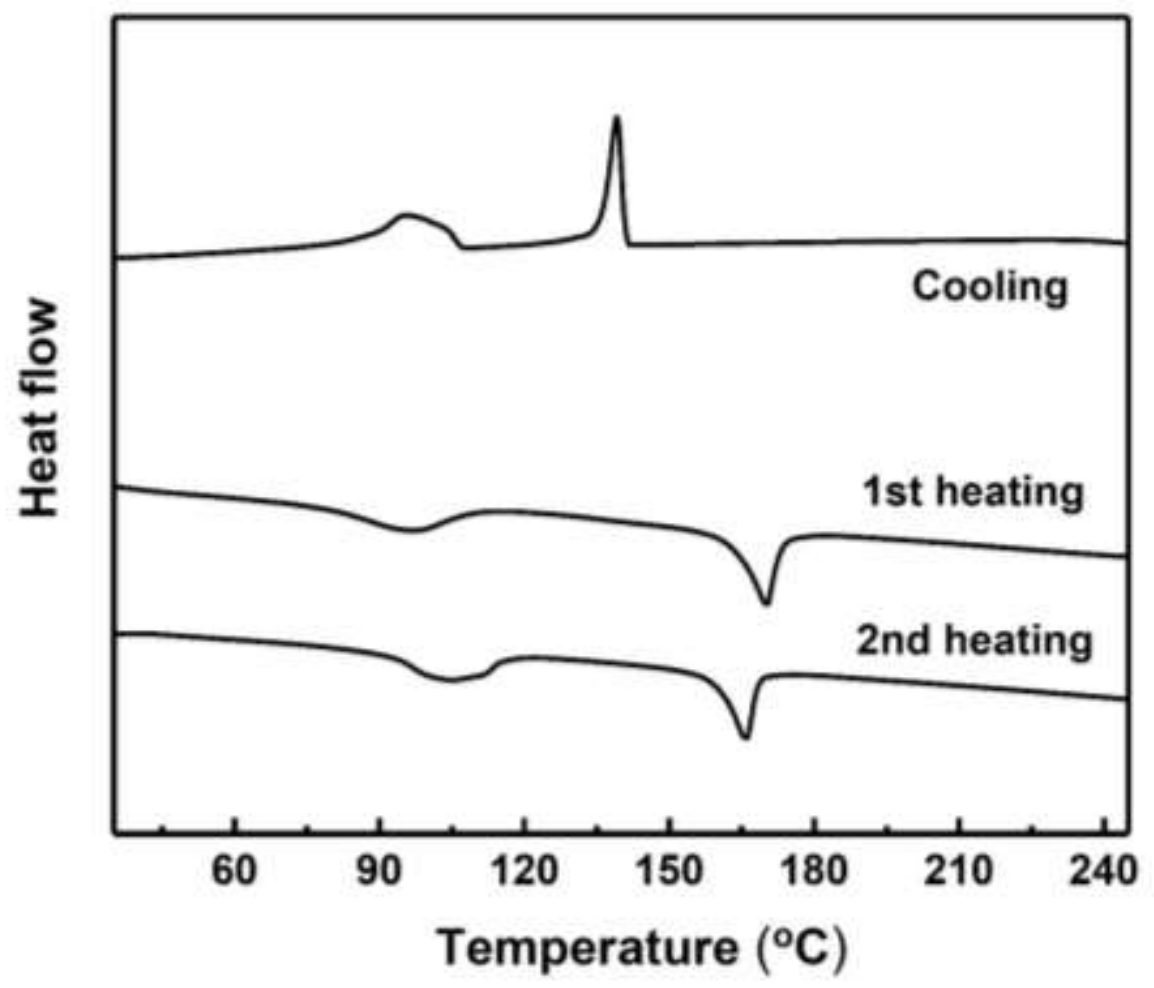

Figure S1. DSC curves of PC12TV12T without SFA side chains 


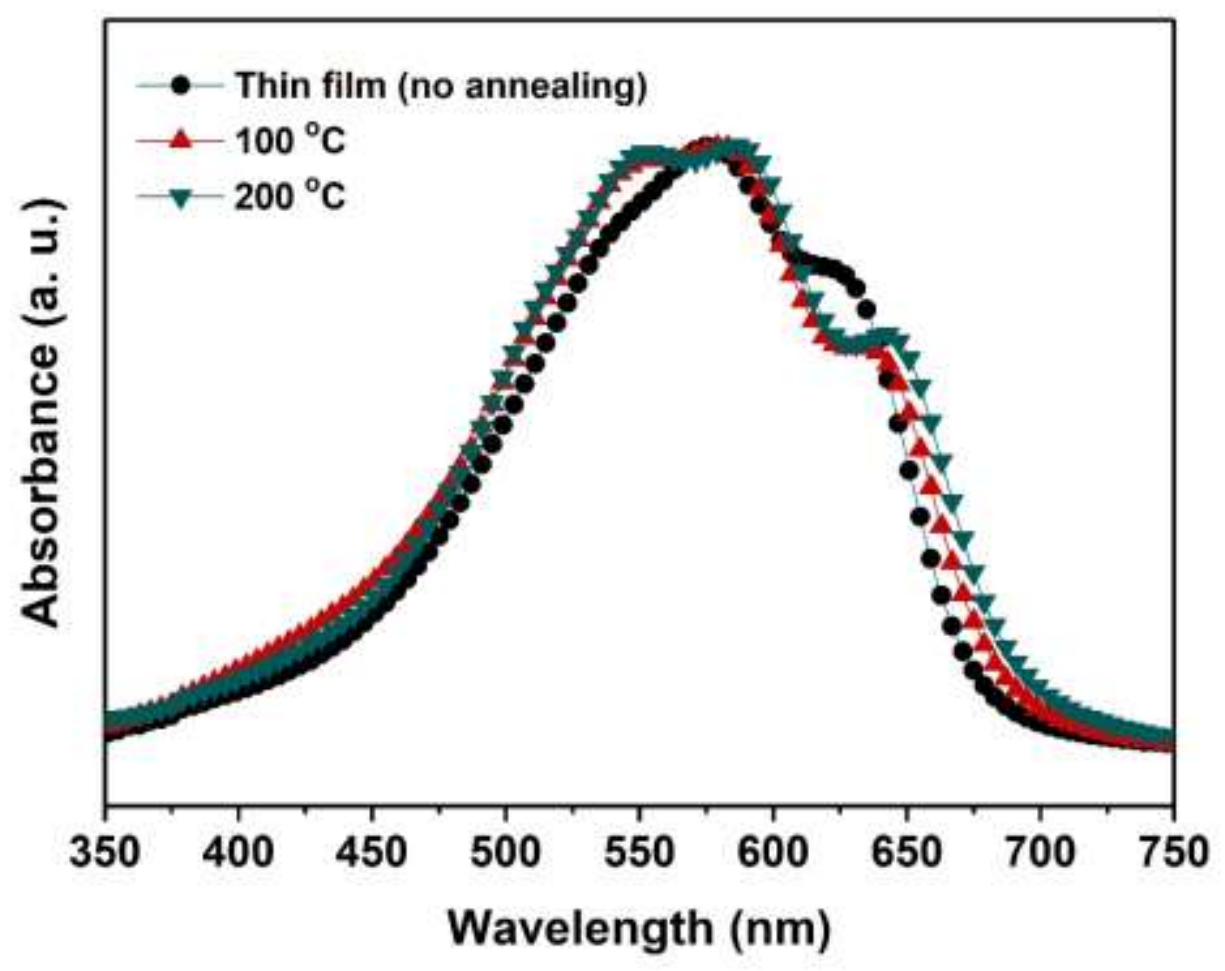

Figure S2. UV-Vis absorption spectra of the PC12TVC5FT thin films depending on annealing temperature. 
No annealing

Contact angle : $109^{\circ}$

Surface energy : $8.21 \mathrm{mN} / \mathrm{m}$

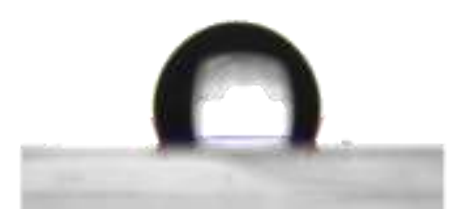

$100{ }^{\circ} \mathrm{C}$

Contact angle : $110^{\circ}$

Surface energy : $7.81 \mathrm{mN} / \mathrm{m}$

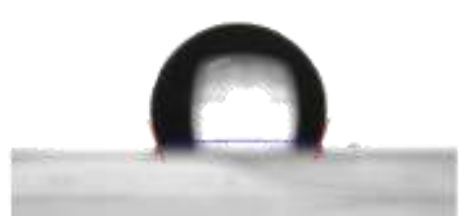

$200{ }^{\circ} \mathrm{C}$

Contact angle : $112^{\circ}$

Surface energy : $7.05 \mathrm{mN} / \mathrm{m}$

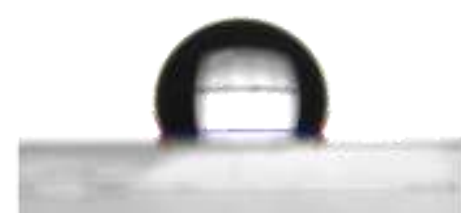

Figure S3. Change of contact angle depending on the annealing temperature of PC12TVC5F7T thin films. 

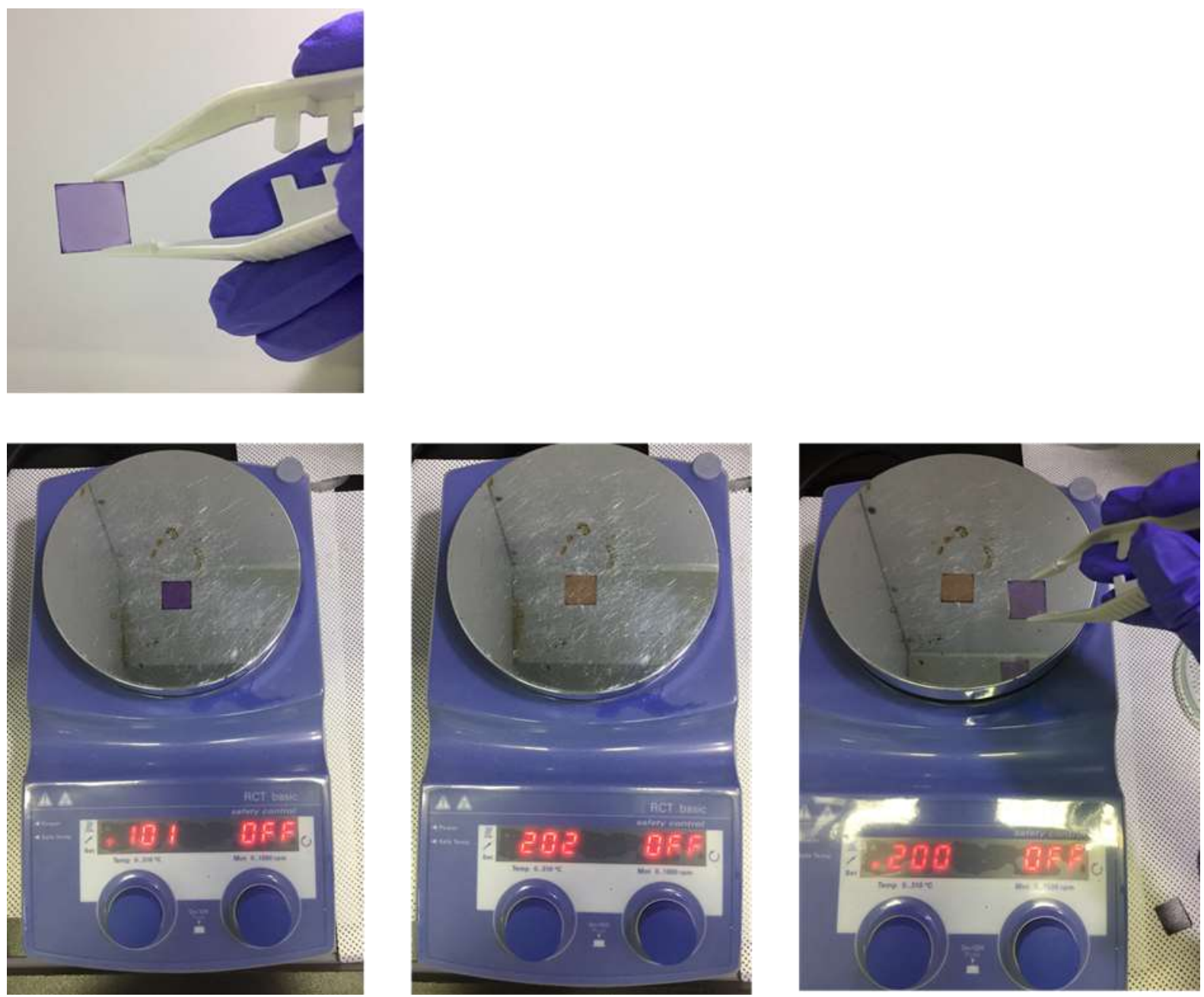

Figure S4. Film images as a function of annealing temperatures. 


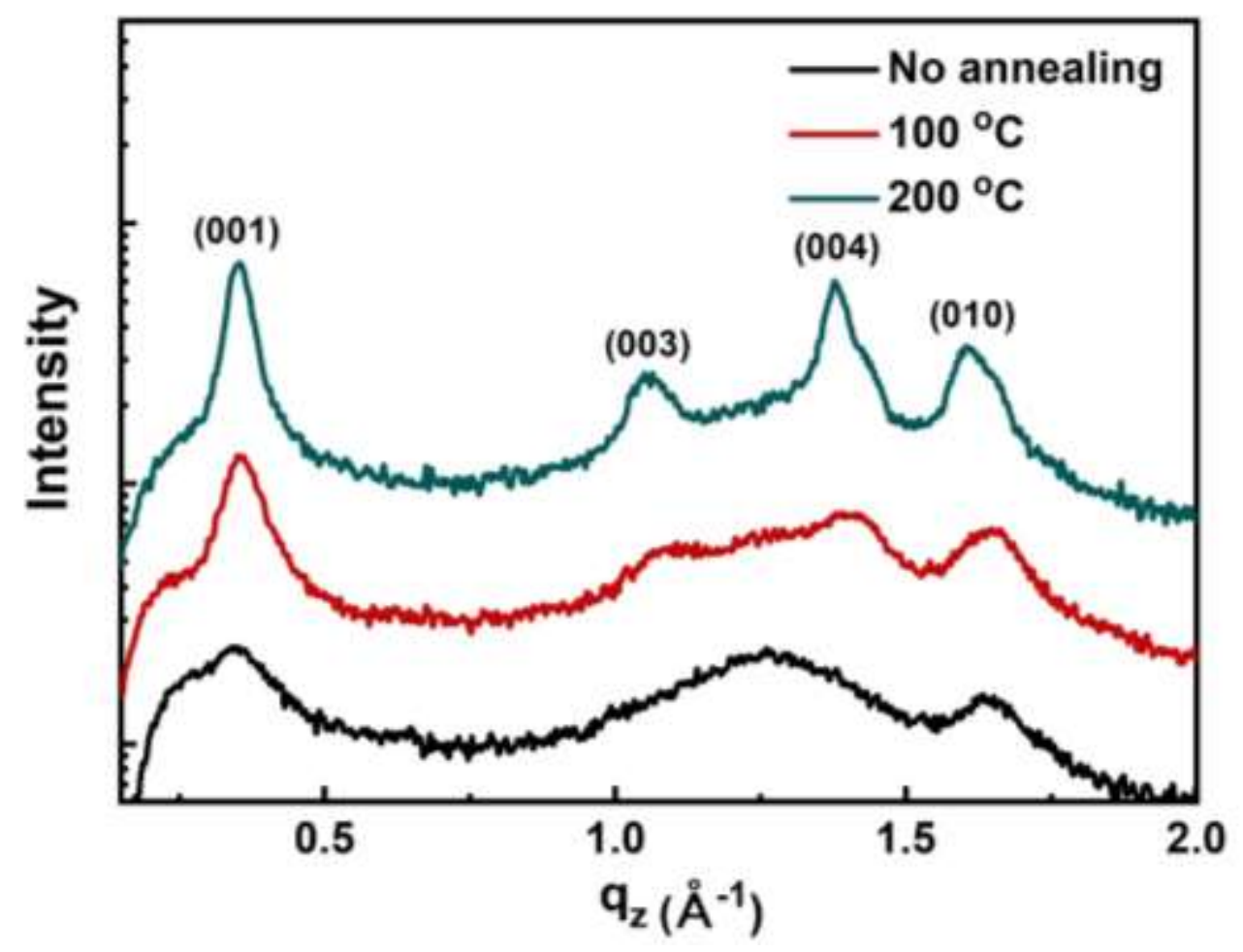

Figure S5. In-plane X-ray diffraction patterns of PC12TVC5F7T. 

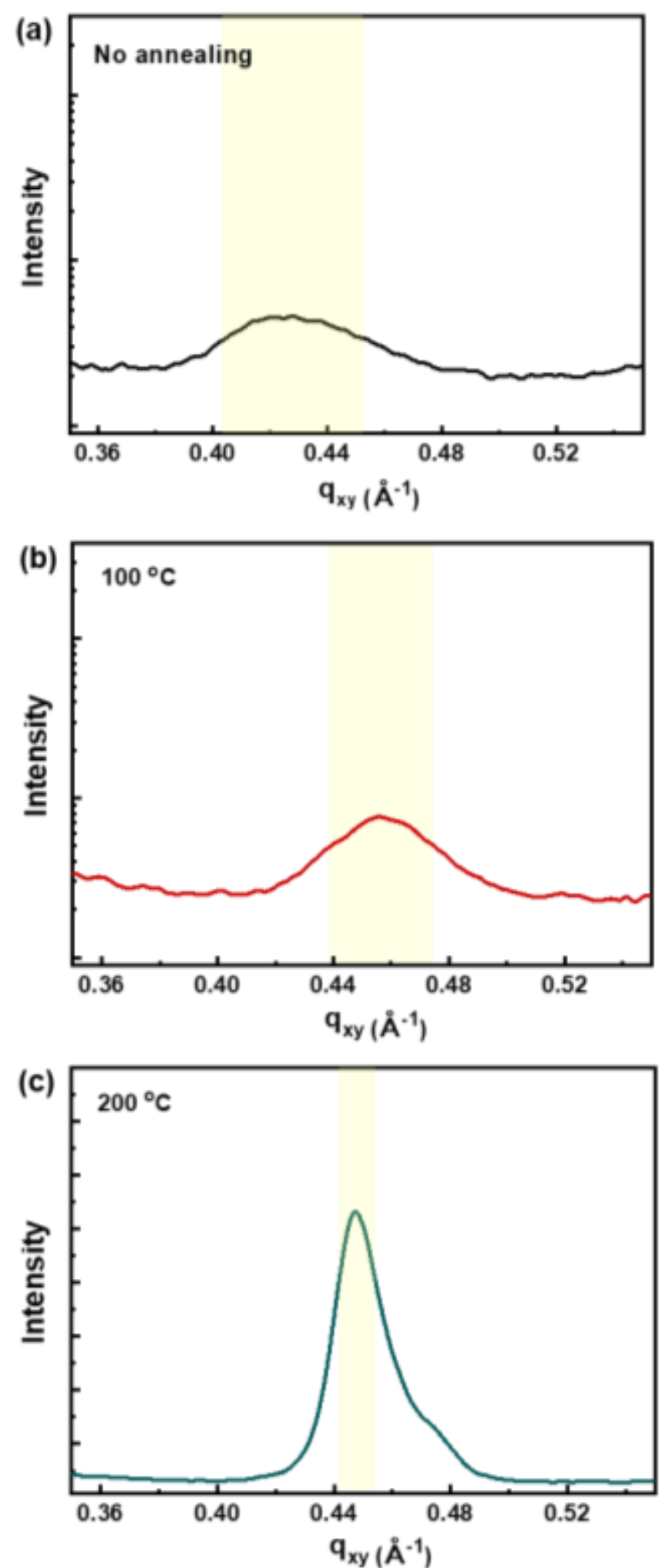

Figure S6. FWHM of (200) peaks in out-of-plane directions for (a) no annealing films, (b) $100{ }^{\circ} \mathrm{C}$ film, and (c) $200{ }^{\circ} \mathrm{C}$ film. 


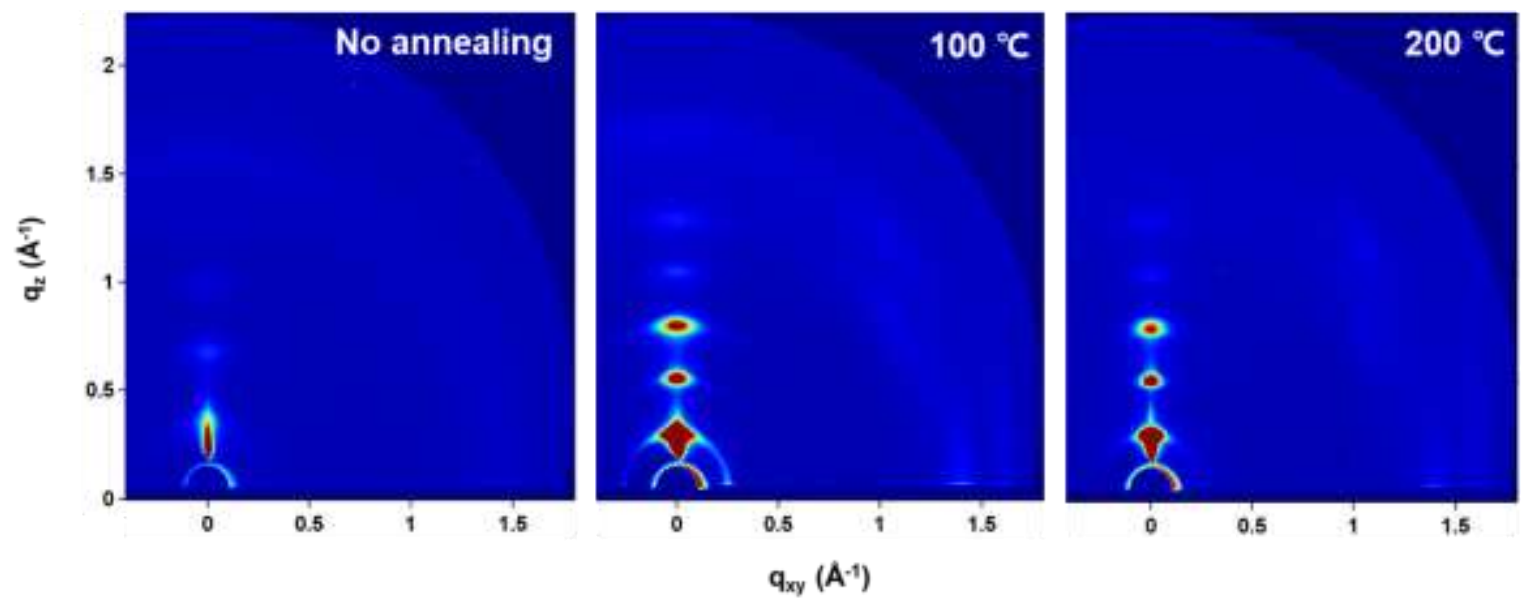

Figure S7. 2D GIWAXS images of PC12TVC12T annealed at different temperatures. 
Table S1 The crystallographic parameters of PC12TVC5FT

\begin{tabular}{|c|c|c|c|c|c|c|c|c|c|c|}
\hline & & \multicolumn{5}{|c|}{ Out of plane } & \multicolumn{4}{|c|}{ In plane } \\
\hline & & (100) & (200) & $(300)$ & $(400)$ & $(500)$ & (001) & (003) & (004) & (010) \\
\hline No annealing & $Q\left(\bar{A}^{-1}\right)$ & 0.214 & 0.419 & 0.634 & - & - & 0.345 & - & - & 1.637 \\
\hline $100{ }^{\circ} \mathrm{C}$ & $Q\left(A^{-1}\right)$ & 0.222 & 0.455 & 0.681 & 0.904 & 1.116 & 0.359 & 1.085 & 1.414 & 1.661 \\
\hline $200{ }^{\circ} \mathrm{C}$ & $Q\left(A^{-1}\right)$ & 0.226 & 0.447 & 0.669 & 0.890 & 1.116 & 0.353 & 1.050 & 1.376 & 1.603 \\
\hline
\end{tabular}



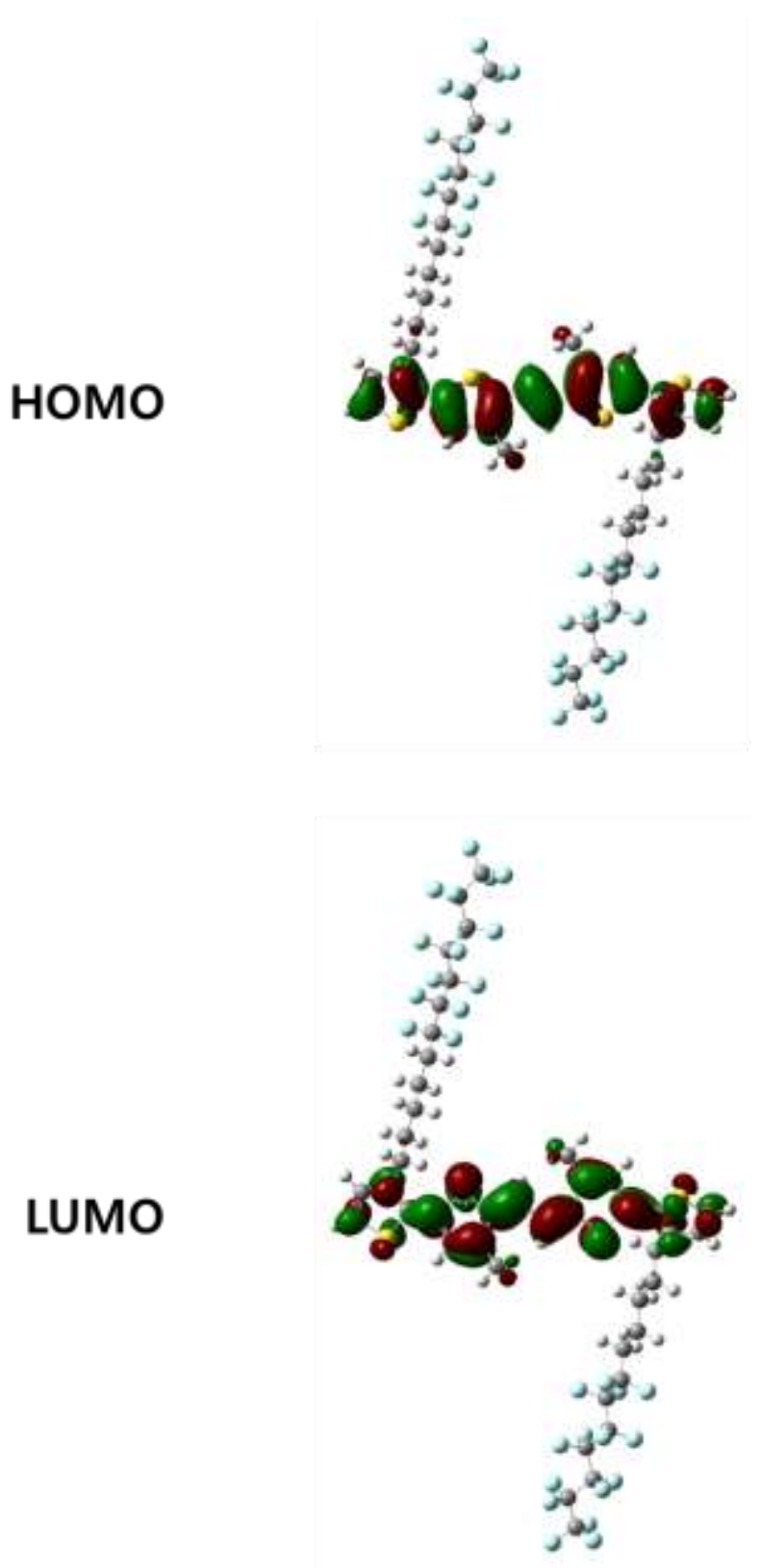

Figure S8. HOMO and LUMO electron density plots of optimized geometries for PC12TVC5F7T 


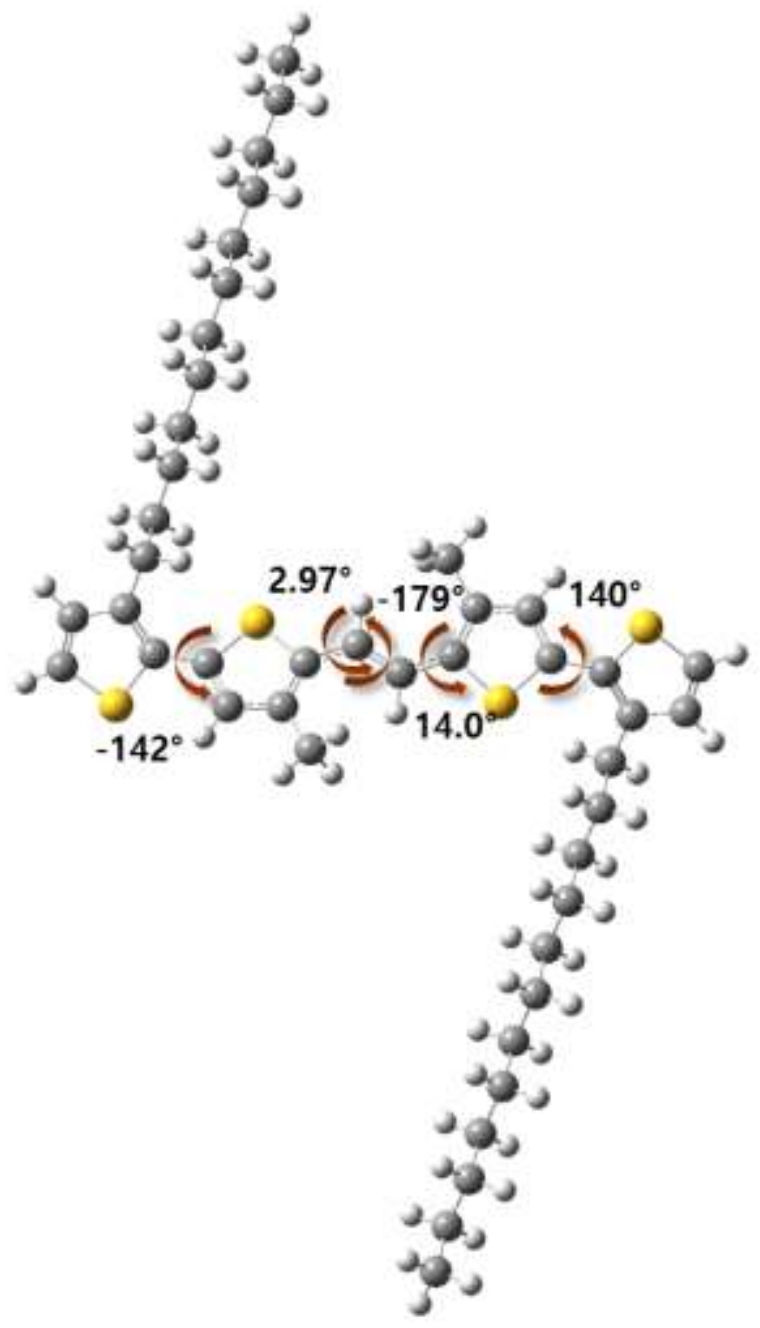

Figure S9. DFT Calculated dihedral angels of PC12TV12T without SFA side chains 


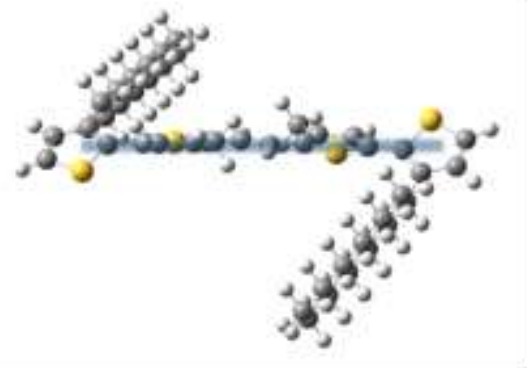

PC12TVC12T

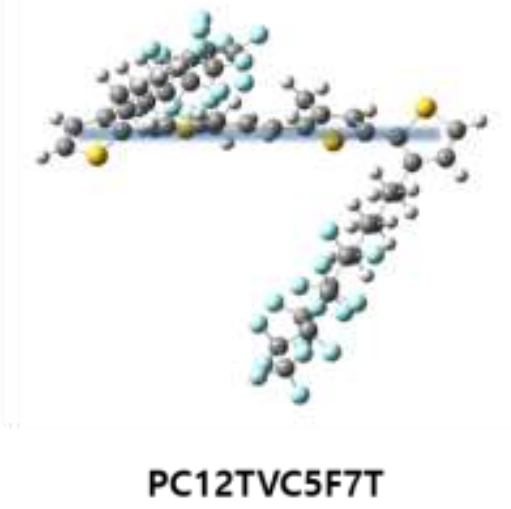

PC12TVC5F7T

Figure S10. Side view of DFT calculations of PC12TV12T and PC12TVC5F7T 